\title{
EXTREMAL PROBLEMS FOR EXTREMAL DISTANCE AND HARMONIC MEASURE
}

\author{
DIMITRIOS BETSAKOS
}

Department of Engineering and Management of Energy Resources, Aristotle University, Kastorias12, Kozani 50100, Greece

E-mail: betsakos@otenet.gr

Abstract: We formulate and solve some geometric extremal problems involving extremal distance and harmonic measure. The extremal configurations are symmetric. We answer a question raised in our earlier work [Bet].

\section{Introduction}

Let $\Omega$ be a simply connected domain in the extended complex plane $\mathbb{C}_{\infty}$ bounded by a Jordan curve $\partial \Omega$. By a well-known theorem of C.Carathéodory every conformal map of $\Omega$ onto another Jordan domain has a continuous oneto-one extension to the closure of $\Omega$; (see e.g. [Oht, §3.1]). Let $E_{1}, E_{2}$ be two disjoint, closed arcs on $\partial \Omega$. The extremal distance $\lambda\left(E_{1}, E_{2}, \Omega\right)$ between $E_{1}$ and $E_{2}$ is defined as follows (cf. [Hen, $\left.§ 16.11\right]$ ):

There is a unique conformal mapping $f: \Omega \rightarrow \mathbb{C}$ and a unique positive number $\lambda$ such that $f$ maps $\Omega$ onto the rectangle $(0, \lambda) \times(0,1)$ with $f\left(E_{1}\right)=$ $\{i y: 0 \leq y \leq 1\}$ and $f\left(E_{2}\right)=\{\lambda+i y: 0 \leq y \leq 1\}$. Then $\lambda\left(E_{1}, E_{2}, \Omega\right):=\lambda$.

There are more general definitions of extremal distance (see [Beu], [Oht]) but here we will consider only the simply connected case described above. Another name for the extremal distance is module of the quadrilateral $\Omega$ with opposite sides $E_{1}, E_{2}$. There is a close relation between extremal distance and harmonic measure. Let $\Omega$ be as above and consider a Borel set $E$ on $\partial \Omega$. The harmonic measure $\omega(z, E, \Omega)$ of $E$, at $z \in \Omega$, with respect to $\Omega$, is defined as follows (cf. [Nev, III §1]):

There is a conformal map $f: \Omega \rightarrow \mathbb{D}=\{z:|z|<1\}$ with $f(z)=0$. Let $\widehat{E}=f(E) \subset \partial \mathbb{D}$. Then $\omega(z, E, \Omega):=|\widehat{E}| /(2 \pi)$, where $|\widehat{E}|$ is the length of $\widehat{E}$.

Date: February 1, 2000.

1991 Mathematics Subject Classification. Primary 30C85, 31A15.

Key words and phrases. Extremal distance, harmonic measure.

This research was partially supported by a grant from the Greek State Foundation of Scholarships (I.K.Y.). 
Both extremal distance and harmonic measure are invariant under conformal mappings. For other properties, we refer to [Beu], [Hen], [Nev], [Oht]. We will study some extremal problems that involve these two conformal invariants and have a simple geometric character. These problems are related to our earlier work [Bet]. In fact, we answer here a uniqueness question which was left open in [Bet]. In the next section we present some auxiliary results. In Sections 3 and 4 we study two problems for extremal distance and in Section 5 we deal with the analogous problems for harmonic measure.

\section{Some auxiliary results}

In some cases extremal distances and harmonic measures can be computed explicitly with the help of conformal maps. Such a computation can be done for the strip:

Let $S_{o}=\{z:-y<\Re z<y\}$ with $y>0$. Let also $A_{o}=\{z: \Re z=$ $-y,|\Im z| \leq a\}, a>0$. Then (see e.g. [Oht, $\S 1.10]$ )

$$
\omega\left(0, A_{o}, S_{o}\right)=\frac{2}{\pi} \arctan \left(e^{\pi a /(2 y)}\right)-\frac{1}{2} .
$$

For $x<0<y$ and $a, b>0$, let $S=\{z: x<\Re z<y\}, A=\{z: \Re z=$ $x,|\Im z| \leq a\}, B=\{z: \Re z=y,|\Im z| \leq b\}$. Then (see e.g. [Bet, Proposition 2.10])

$$
\lambda(A, B, S)=\left[4 \nu\left(\frac{X+Y}{1+X Y}\right)\right]^{-1},
$$

where $X=\exp \left(\frac{-\pi a}{y-x}\right), Y=\exp \left(\frac{-\pi b}{y-x}\right)$, and $\nu$ is the function defined by

$$
\begin{gathered}
\nu(r)=\frac{1}{4} \frac{\mathcal{K}^{\prime}(r)}{\mathcal{K}(r)}, \quad \mathcal{K}(r)=\int_{0}^{1} \frac{d t}{\left(1-t^{2}\right)^{1 / 2}\left(1-r^{2} t^{2}\right)^{1 / 2}}, \\
\mathcal{K}^{\prime}(r)=\mathcal{K}\left(r^{\prime}\right), \quad r^{\prime}=\sqrt{1-r^{2}}, \quad r \in(0,1) .
\end{gathered}
$$

The function $\nu$ plays an important role in the theory of quasiconformal mapping and other branches of Analysis (see [AVV]). Sometimes we also use the function $\mu=2 \pi \nu$. We mention two properties of these functions (see $[\mathrm{AVV}$, ch. 5]):

$$
\begin{array}{ll}
\nu(r)=2 \nu\left(\frac{2 \sqrt{r}}{1+r}\right), & 0<r<1, \\
\mu^{\prime}(r)=\frac{-\pi^{2}}{4 r r^{2} \mathcal{K}(r)^{2}}, & 0<r<1 .
\end{array}
$$

Extremal distances on the upper half-plane $\mathbb{H}$ can be also computed explicitly (see [Oht, §2.16]):

$$
\lambda([-a, 0],[b, b+c], \mathbb{H})=4 \nu\left(\sqrt{\frac{a c}{(a+b)(b+c)}}\right) .
$$




\section{An extremal problem for extremal distance}

Fix $0<\theta<\pi / 2$. For $\phi \in[-\theta, \theta)$, let $l_{A}$ be the line passing through the points $-e^{-i \theta}$ and $-e^{-i \phi}$, and $l_{B}$ be the line passing through the points $e^{i \theta}$ and $e^{i \phi}$. Let $D=D(\phi)$ be the component of $\mathbb{C}_{\infty} \backslash\left(l_{A} \cup l_{B}\right)$ that contains the origin. Thus $D$ is an angular domain if $\phi \neq-\theta$, and $D$ is a vertical strip if $\phi=-\theta$. If $\phi \neq-\theta$ the vertex of $D$ is at the point $i \tilde{y}$ with

$$
\tilde{y}=\tilde{y}(\phi)=\sin \theta+\frac{\sin \theta-\sin \phi}{\cos \phi-\cos \theta} \cos \theta>0 .
$$

The angle of $D$ at $i y_{o}$ is

$$
\psi=\psi(\phi)=2 \arctan \left(\frac{\cos \phi-\cos \theta}{\sin \theta-\sin \phi}\right) \in(0, \pi) .
$$

Let $A=A(\phi):=l_{A} \cap \mathbb{D}, B=B(\phi):=l_{B} \cap \mathbb{D}$. We will solve the following extremal problem.

Problem 1. Find $\min _{\phi \in[-\theta, \theta)} \lambda(A, B, D)$ and determine the extremal configuration(s).
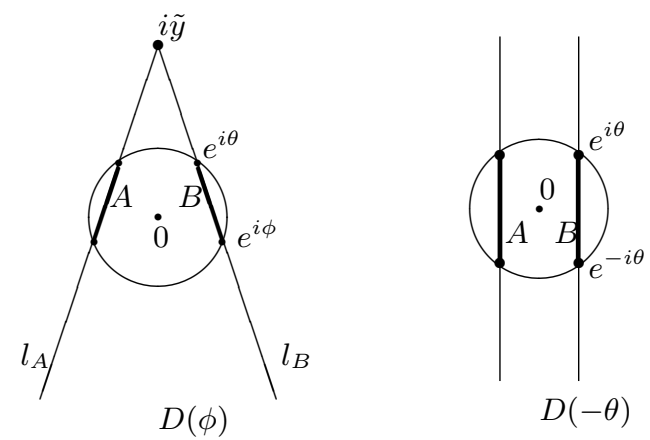

Figure 1: A competing domain $D(\phi), \phi \neq-\theta$ and the extremal domain $D(-\theta)$.

Theorem 1. With the above notation

$$
\min _{\phi \in[-\theta, \theta)} \lambda(A, B, D)=\left[2 \nu\left(e^{-\pi \tan \theta}\right)\right]^{-1} .
$$

The minimum is attained uniquely for $\phi=-\theta$.

Proof. We compute $\lambda(A, B, D)$ explicitly as a function of $\phi$. A computation based on (2.2) and (2.3) shows that

$$
\lambda(A(-\theta), B(-\theta), D(-\theta))=\left[2 \nu\left(e^{-\pi \tan \theta}\right)\right]^{-1} .
$$

Assume next that $\phi \neq-\theta$. The angular domain $D$ is a rotation of the domain

$$
\widehat{D}=\left\{r e^{i \xi}: r>0,0<\xi<\psi\right\} .
$$


A simple geometric computation yields

$$
\begin{aligned}
& l_{1}:=\left|i \tilde{y}-e^{i \theta}\right|=\frac{\cos \theta}{\sin (\psi / 2)}, \\
& l_{2}:=\left|i \tilde{y}-e^{i \phi}\right|=\frac{\cos \phi}{\sin (\psi / 2)} .
\end{aligned}
$$

The length of $A$ (and of $B$ ) is equal to $l_{2}-l_{1}$.

The function $z \rightarrow z^{\pi / \psi}$ maps $\widehat{D}$ conformally onto the upper half-plane $\mathbb{H}$. The conformal invariance of extremal distance implies that

$$
\lambda(A, B, D)=\lambda\left(\left[-l_{2}^{\pi / \psi},-l_{1}^{\pi / \psi}\right],\left[l_{1}^{\pi / \psi}, l_{2}^{\pi / \psi}\right], \mathbb{H}\right) .
$$

The latter extremal distance can be computed explicitly in terms of complete elliptic integrals: Using (2.5) we find

$$
\lambda\left(\left[-l_{2}^{\pi / \psi},-l_{1}^{\pi / \psi}\right],\left[l_{1}^{\pi / \psi}, l_{2}^{\pi / \psi}\right], \mathbb{H}\right)=4 \nu\left(\frac{l_{2}^{\pi / \psi}-l_{1}^{\pi / \psi}}{l_{2}^{\pi / \psi}+l_{1}^{\pi / \psi}}\right) .
$$

It is well-known that the function $\nu$ is strictly decreasing and continuous on $(0,1)$; see e.g. [AVV, Ch. 5]. Hence $\lambda(A, B, D)$, as a function of $\phi$, is continuous for $\phi \in(-\theta, \theta)$. Moreover, it is continuous (from the right) at $\phi=-\theta$. This fact can be easily proved using a simple covergence argument, (2.2) and (2.3). Therefore, in order to prove the theorem, we must prove the following

Claim: The quantity $\left(l_{2}^{\pi / \psi}-l_{1}^{\pi / \psi}\right) /\left(l_{2}^{\pi / \psi}+l_{1}^{\pi / \psi}\right)$ as function of $\phi$ is strictly decreasing.

Proof of the claim: Substituting $l_{1}, l_{2}$ from (3.5), (3.6), we find

$$
\frac{l_{2}^{\pi / \psi}-l_{1}^{\pi / \psi}}{l_{2}^{\pi / \psi}+l_{1}^{\pi / \psi}}=\frac{(\cos \phi / \cos \theta)^{\pi / \psi}-1}{(\cos \phi / \cos \theta)^{\pi / \psi}+1}
$$

Define

$$
f(\phi):=(\cos \phi / \cos \theta)^{\pi / \psi}=\exp \left(\frac{\pi \log \frac{\cos \phi}{\cos \theta}}{2 \arctan \left(\frac{\cos \phi-\cos \theta}{\sin \theta-\sin \phi}\right)}\right) .
$$

The second equality above comes from (3.2). It is easy to see that to prove the claim, it suffices to prove that $f$ is strictly decreasing. Let

$$
g(\phi):=\frac{\log \frac{\cos \phi}{\cos \theta}}{\arctan \left(\frac{\cos \phi-\cos \theta}{\sin \theta-\sin \phi}\right)}
$$

We prove that $g$ is strictly decreasing for $\phi \in(-\theta, \theta)$ :

A straightforward calculation shows that

$$
\left[\arctan \left(\frac{\cos \phi-\cos \theta}{\sin \theta-\sin \phi}\right)\right]^{\prime} \equiv \frac{1}{2}
$$


Thus $g^{\prime}(\phi)<0$ if and only if

$$
s(\phi):=-\tan \phi \arctan \left(\frac{\cos \phi-\cos \theta}{\sin \theta-\sin \phi}\right)-\frac{1}{2} \log \frac{\cos \phi}{\cos \theta}<0 .
$$

But $s(-\theta)=0$ and also by (3.9),

$$
s^{\prime}(\phi)=-\frac{\tan \phi}{2}-\frac{1}{\cos ^{2} \phi} \arctan \left(\frac{\cos \phi-\cos \theta}{\sin \theta-\sin \phi}\right)+\frac{\tan \phi}{2}<0 .
$$

Hence $g$ is strictly decreasing. This implies that $f$ is also strictly decreasing and the claim is proved. The proof of the theorem is also complete now.

\section{Another extremal problem for extremal distance}

In this section we study a problem which involves extremal and euclidean distances. It may be considered as an illustration of the interplay between conformal and euclidean quantities studied in the book of R.Nevanlinna [Nev, ch. IV].

Let $x \in[-1,0], y \in(x, 1]$, and $S=S(x, y):=\{z: x<\Re z<y\}$. Let also

$$
\begin{gathered}
E=E(x):=\partial S \cap \mathbb{D} \cap\{z: \Re z=x\}, \\
F=F(y):=\partial S \cap \mathbb{D} \cap\{z: \Re z=y\} .
\end{gathered}
$$

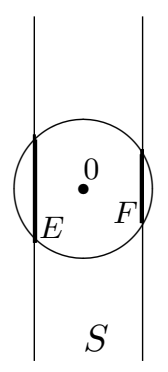

Figure 2: The vertical segments $E$ and $F$ on $\partial S$.

Define

$$
\Lambda(x, y):=\lambda(E, F, S)
$$

The extremal problem we will study is:

Problem 2. Find

$$
\min _{x, y} \frac{\Lambda(x, y)}{y-x}
$$

and determine all the extremal configuration(s).

This problem was studied in [Bet, Section 5] where we have shown:

Fact (a): The minimum is attained for at least one pair $(x, y)$.

Fact (b): If $(x, y)$ is a minimizing pair then $y=-x \in(0,1)$. 
Fact (c): The function $\Lambda$ has the following explicit expression in terms of elliptic integrals (cf. (2.2)):

$$
\Lambda(x, y)=\left[4 \nu\left(\frac{X+Y}{1+X Y}\right)\right]^{-1}
$$

where

$X=X(x, y):=\exp \left(\frac{-\pi \sqrt{1-x^{2}}}{y-x}\right), \quad Y=Y(x, y):=\exp \left(\frac{-\pi \sqrt{1-y^{2}}}{y-x}\right)$.

Here we will complete the solution of Problem 2 by proving the following theorem.

Theorem 2. There is a unique number $y_{0} \in(0,1)$ such that

$$
\min _{x, y} \frac{\Lambda(x, y)}{y-x}=\frac{\Lambda\left(-y_{o}, y_{o}\right)}{2 y_{o}} .
$$

Proof. It remains only to prove that the minimum is attained uniquely. Because of Fact (b) above, it suffices to prove that the

$$
\min _{y \in(0,1)} \frac{\Lambda(-y, y)}{2 y}
$$

is attained uniquely for some $y_{o} \in(0,1)$.

In view of Fact (c), we consider the function

$$
l(y)=\left[8 y \nu\left(\frac{2 \exp \left(-\pi \sqrt{1-y^{2}} /(2 y)\right)}{1+\exp \left(-\pi \sqrt{1-y^{2}} / y\right)}\right)\right]^{-1}, \quad y \in(0,1) .
$$

We must prove that $l$ attains its minimum uniquely. We will use the function $\mu=2 \pi \nu$ instead of the function $\nu$ in order to be in agreement with the notation of $[\mathrm{AVV}]$. With this notation and by (2.3), we have

$$
l(y)=\frac{\pi}{4 y \mu\left(\frac{2 \exp \left(-\pi \sqrt{1-y^{2}} /(2 y)\right)}{1+\exp \left(-\pi \sqrt{1-y^{2}} / y\right)}\right)}=\frac{\pi}{2 y \mu\left(e^{-\pi \sqrt{1-y^{2}} / y}\right)} .
$$

We do the change of variable

$$
r=e^{-\pi \sqrt{1-y^{2}} / y},
$$

or equivalently

and consider the function

$$
y=\frac{\pi}{\sqrt{\pi^{2}+\log ^{2} r}},
$$

$$
L(r)=\frac{\sqrt{\pi^{2}+\log ^{2} r}}{2 \mu(r)} .
$$

The transformation $r=r(y):(0,1) \rightarrow(0,1)$ is strictly increasing. Hence if $L$ attains uniquely its minimum at some point $r_{o}$ then $l$ attains uniquely its minimum for some point $y_{o}$. 
We differentiate the function $L$ :

$$
L^{\prime}(r)=\frac{\mu(r) \log r-r \mu^{\prime}(r)\left(\pi^{2}+\log ^{2} r\right)}{2 r \mu(r)^{2} \sqrt{\pi^{2}+\log ^{2} r}} .
$$

Hence we must prove that the equation

$$
\mu(r) \log r=r \mu^{\prime}(r)\left(\pi^{2}+\log ^{2} r\right)
$$

has a unique solution in $(0,1)$. We use $(2.4)$ and after some simple calculations, (4.1) becomes

$$
\frac{2}{\pi} r^{\prime 2} \mathcal{K}(r) \mathcal{K}^{\prime}(r)=\left(\pi^{2}+\log ^{2} r\right) \log \frac{1}{r}
$$

or equivalently

$$
\frac{m(r)}{\log \frac{1}{r}}=\pi^{2}+\log ^{2} r
$$

where $m(r)=(2 / \pi) r^{\prime 2} \mathcal{K}(r) \mathcal{K}^{\prime}(r)$.

But by [AVV, Theorem 3.28], the function $m(r) / \log (1 / r)$ is strictly increasing from $(0,1)$ to $(1, \infty)$. Since the function $\pi^{2}+\log ^{2} r$ is strictly decreasing from $(0,1)$ to $\left(\pi^{2}, \infty\right)$, the equation (4.2) (and hence the equation (4.1) too) has a unique solution in $(0,1)$. This fact finishes the proof of the theorem.

\section{Remarks}

1. As it was noted in [Bet], the numerical value of $y_{o}$ is approximately equal to 0.403 . It was mentioned there that perhaps $y_{o}=\cos \frac{\pi}{e}$, but more accurate numerical computations show that this is not true.

2. Since Problem 4 has a unique solution, the problem for harmonic measure studied in [Bet, Section 8] has also a unique solution.

3. One can combine Problems 1 and 2 , and show that the strip $S\left(-y_{0}, y_{o}\right)$ is the unique extremal domain for a more general extremal problem.

\section{Extremal problems for harmonic measure}

In this section we study two extremal problems for harmonic measure that are analogous with the Problems 1 and 2.

5.1. The first problem is associated to the configuration of Problem 1 . We will use the notation $\theta, \phi, D=D(\phi), A=A(\phi), B=B(\phi), \lambda(A, B, D)$ of Section 3.

Problem 3. Find $\max _{\phi \in[-\theta, \theta)} \omega(0, A \cup B, D)$ and determine the extremal configuration(s).

Theorem 3. With the above notation

$$
\max _{\phi \in[-\theta, \theta)} \omega(0, A \cup B, D)=\frac{4}{\pi} \arctan \left(e^{\frac{\pi}{2} \tan \theta}\right)-1 .
$$

The maximum is attained uniquely for $\phi=-\theta$. 
The harmonic measure $\omega(0, A \cup B, D)$ can be written explicitly in terms of elementary functions. Such an expression, however, is complicated and it is difficult to prove Theorem 3 using it. We will apply a different method that uses Theorem 1 and exploits the symmetry of $D$ and the additional symmetry of $D(-\theta)$.

Proof. Let $\lambda=\lambda(\phi):=\lambda(A, B, D)$, and let $f$ map $D$ conformally onto the rectangle $R=R(\phi):=(0, \lambda) \times(0,1)$ with $\widehat{A}:=f(A)=\{i y: 0 \leq y \leq 1\}$ and $\widehat{B}:=f(B)=\{\lambda+i y: 0 \leq y \leq 1\}$. Because of symmetry we have

$$
\widehat{O}:=f(0)=\frac{\lambda}{2}+i t
$$

for some $t \in(0,1)$.

By the conformal invariance of harmonic measure,

$$
\omega(0, A \cup B, D)=\omega(\widehat{O}, \widehat{A} \cup \widehat{B}, R) .
$$

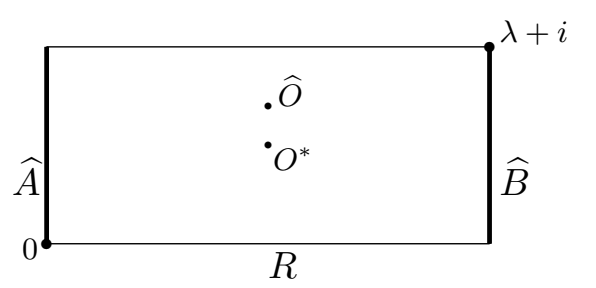

Figure 3: The rectangle $R$ with opposite sides $\widehat{A}, \widehat{B}$.

Now Theorem 3 follows at once from the following four facts:

(i): For all $\phi \in(-\theta, \theta)$,

$$
\lambda(\phi)>\lambda(-\theta)
$$

this is Theorem 1 .

(ii): For all $\phi \in[-\theta, \theta)$,

$$
\omega(\widehat{O}, \widehat{A} \cup \widehat{B}, R) \leq \omega\left(O^{*}, \widehat{A} \cup \widehat{B}, R\right),
$$

where $O^{*}=\lambda / 2+i / 2$; this comes (for instance) from a classical symmetrization result for harmonic measure [Hal].

(iii): For all $\phi \in[-\theta, \theta)$,

$$
\omega(\lambda(\phi) / 2+i / 2, \widehat{A} \cup \widehat{B}, R(\phi)) \leq \omega(\lambda(-\theta) / 2+i / 2, \widehat{A} \cup \widehat{B}, R(-\theta)) ;
$$

this is an immediate consequence of (i) and the maximum principle.

(iv): For $\phi=-\theta, D(-\theta)$ is a strip and we have

$$
\omega(0, A(-\theta), B(-\theta), D(-\theta))=\frac{4}{\pi} \arctan \left(e^{\frac{\pi}{2} \tan \theta}\right)-1
$$

this follows from a simple computation based on (2.1). 
5.2. Finally we study a problem for harmonic measure associated to the configuration of Section 4. We use the notation $x, y, E=E(x), F=F(y)$, $S=S(x, y)$ of Section 4 .

Problem 4. Find $\max _{x, y}(y-x) \omega\left(\frac{x+y}{2}, E \cup F, S\right)$ and determine the extremal configuration(s).

The solution of this problem is similar to the solution of Problem 2. Here we only outline the solution and leave the details to the reader.

We first express $\omega((x+y) / 2, E \cup F, S)$ as function of $x, y$ using (2.1). This expression involves only trigonometric and other elementary functions (and not elliptic integrals). Following the techniques of [Bet, Section 5] and of Section 4 we prove the following facts:

- The maximum is attained at least for one pair $(x, y)$ with $0<x<$ $1,0<y<1$.

- If $(x, y)$ is a maximizing pair then $y=-x$.

- The function $\omega(y)=2 y \omega(0, E(-y) \cup F(y), S(-y, y))$ attains uniquely its maximum at some point $y^{*} \in(0,1)$.

- The numerical value of $y^{*}$ is approximately equal to 0.6935 .

We summarize the results on Problem 4:

Theorem 4. The maximum of $(y-x) \omega\left(\frac{x+y}{2}, E \cup F, S\right)$ is attained uniquely for a pair $\left(-y^{*}, y^{*}\right)$ with $y^{*} \approx 0.6935$.

Remark. In Problems 1,2,3,4 the unique extremal domains have additional symmetries in comparison with the competing domains.

\section{REFERENCES}

[AVV] G.D.Anderson, M.K.Vamanamurthy, and M.Vuorinen, Conformal Invariants, Inequalities, and Quasiconformal Maps, Wiley, New York 1997.

[Bet] D.Betsakos, Harmonic measure on simply connected domains of fixed inradius. Ark. Mat. 36 (1998), 275-306.

[Beu] A.Beurling, Extremal distance and estimates for harmonic measure, pp. 361-385 in 'The collected works of Arne Beurling, vol.1 Complex Analysis', ed. by L.Carleson et al. Birkhäuser, Boston 1989.

[Hal] K.Haliste, Estimates of harmonic measures. Ark. Mat. 6 (1965), 1-31.

[Hen] P.Henrici, Applied and Computational Complex Analysis, vol. III, Wiley, New York 1986.

[Nev] R.Nevanlinna, Analytic Functions. Springer, New York 1970 (first edition in German 1936).

[Oht] M.Ohtsuka, Dirichlet Problem, Extremal Length and Prime Ends. Van Nostrand, New York 1970. 